\title{
Diagnóstico de la Condición de las Barras de Motores de Inducción
}

\author{
Christian E. Talbot ${ }^{(1)}$, Pedro N. Saavedra ${ }^{(1)}$, M. Aníbal Valenzuela ${ }^{(2)}$ \\ Universidad de Concepción, (1) Departamento de Ingeniería Mecánica, (2) Departamento de Ingeniería \\ Eléctrica, Edmundo Larenas, Concepción-Chile (e-mail: ctalbot@udec.cl)
}

Recibido Ene. 11, 2013; Aceptado Feb. 21, 2013; Versión final recibida Abr. 24, 2013

\begin{abstract}
Resumen
El objetivo del presente trabajo es comparar tres técnicas que permiten diagnosticar barras dañadas en un motor de inducción. Ellas son el análisis de la corriente, el análisis de la velocidad angular instantánea y el análisis de las vibraciones. Se realiza un análisis teórico y una revisión crítica de la literatura donde se analizan las tres técnicas. La convalidación experimental de ellas se realiza en bancos de ensayos. Se daña ex profeso una barra desde un pequeño valor hasta cortarla totalmente y se evalúa el desempeño de cada técnica en la detección del problema. El análisis de las vibraciones mecánicas demostró requerir un nivel de daño en las barras considerablemente mayor que las otras dos técnicas para poder detectar el problema. Se concluye que la técnica dela velocidad angular instantánea fue la más efectiva de las tres, ya que fue capaz de detectar daños incipientes tan pequeños como porosidades en las barras.
\end{abstract}

Palabras clave: motor de inducción, barras dañadas, análisis de vibraciones, análisis de corriente, velocidad angular instantánea (VAl)

\section{Diagnosis of Induction Motors Bar Condition}

\begin{abstract}
The aim of this study is to compare three techniques to diagnose damaged bars in a squirrel cage rotor. They are the current analysis, the instantaneous angular speed analysis and vibration analysis. A theoretical and bibliographical research was conducted where the three techniques were studied. The experimental validation is performed on test benches. A rotor bar is expressly damaged from a small value to completely cut, and then each technique performance in the detection of the problem was evaluated. The mechanical vibration analysis showed to require a substantially higher damage level than the two other techniques to detect the problem. In this case, the technique based on instantaneous angular speed showed to be the most incipient of the three, as it was able to detect damage as small as porosities in the bars.
\end{abstract}

Keywords: induction motor, damaged bars, vibration analysis, current analysis, Instantaneous angular speed (IAS) 


\section{INTRODUCCIÓN}

Desde el punto de vista de los motores de inducción, se han hecho grandes avances a lo largo de los años en relación a la construcción y manufactura de sus respectivos estatores, por lo que éstos presentan cada vez menos fallas en relación a sus respectivos rotores, específicamente los del tipo jaula de ardilla. Así, el porcentaje de fallas que incluye el daño de las barras o anillos se ha incrementado y ha tomado mayor importancia (Nandi y Toliyat, 1999).

De manera general, un motor eléctrico puede presentar problemas eléctricos y problemas mecánicos. Esto dependiendo si tienen relación directa con el circuito eléctrico que conforma la máquina o no. En el presente trabajo se estudiarán tres técnicas para detectar la presencia de barras dañadas en el rotor de un motor de inducción. Este problema se encuentra dentro de la categoría de los problemas de origen eléctrico, debido a que una barra dañada distorsiona las corrientes que fluyen dentro del motor.

Según Szabó et al. (2004), apoyados en la investigación de Nandi y Toliyat (1999) existen numerosos métodos para diagnosticar el problema de las barras dañadas, sin embargo indican que el más usado y que entrega mejores resultados es el análisis de la corriente que alimenta al estator. Si bien este método es simple y fácil de implementar, Sasi et al. (2006) indican en su trabajo que el diagnóstico basado en el análisis de las variaciones de la Velocidad Angular Instantánea (VAI) en el eje es más incipiente que la técnica tradicional de la corriente, sin embargo existen algunas suposiciones y resultados en ese trabajo que ameritan un análisis más exhaustivo.

La idea de utilizar este método en vez del método convencional del análisis de la corriente es poder detectar el daño en una barra lo más incipiente posible. Sasi et al (2006) sin embargo, validan experimentalmente el modelo utilizando un motor con una barra completamente rota en el rotor. Bajo estas condiciones, la falla puede ser detectada por el análisis de corriente y por lo tanto, no existe una clara ventaja del uso de este método. Otra crítica a dicho trabajo es la hipótesis utilizada que considera que la variación del par resistente es función de la velocidad de rotación. Esta hipótesis permitió que las simulaciones numéricas llevadas a cabo en ese trabajo coincidieran con los resultados experimentales.

El objetivo de este trabajo es comparar tres técnicas para diagnosticar barras dañadas en el rotor de un motor de inducción y establecer cuál de ellas es capaz de detectar el problema de manera más incipiente. Estas técnicas son el análisis de la corriente que alimenta al estator, el análisis de la Velocidad Angular Instantánea y el análisis de las vibraciones mecánicas.

\section{ANÁLISIS DE LA CORRIENTE QUE ALIMENTA AL ESTATOR}

El método del análisis de la corriente consiste básicamente en medir la forma de onda de la corriente en cualquiera de las tres fases del motor y luego calcular su espectro. Para medir la corriente, se utiliza un sensor que consiste en un toroide que mide el efecto electromagnético de la corriente que pasa a través de él, entregando una señal de voltaje proporcional a la magnitud de esta corriente.

El análisis de la corriente es utilizado también en la detección de problemas en el motor, como para el caso en que un rodamiento está dañado por una picadura en su pista externa. En este caso, cada vez que un elemento rodante pase por la falla, el motor requerirá un leve aumento de corriente. Así, se tendrá que la corriente estará modulada en amplitud a la frecuencia en que los elementos rodantes pasan por la falla, llamada BPFO (Ball Passing Frequency of Outer race), descrita por Roque et al. (2008). Fig. 1 a) muestra la forma de la corriente en una fase del motor para el caso en que el motor no tiene fallas, la fuente del voltaje de alimentación es sinusoidal y que el circuito tiene un comportamiento lineal. Fig. 1 b) muestra la forma de la corriente para el caso del rodamiento dañado. Fig. 2 muestra el espectro de la corriente para el caso en que existe un rodamiento dañado en su pista externa.

En general, eventos que generen variaciones en el par resistente, modularán la corriente. Estos eventos pueden ser variados, incluyendo variaciones de la carga en el proceso, defectos en rodamientos como daño en las pistas o elementos rodantes o desalineamientos de los ejes a través de los acoplamientos. Estas modulaciones, aunque pequeñas, pueden ser detectadas en el espectro de la corriente con escala logarítmica o expresada en decibeles, presentándose como bandas laterales si esta modulación es periódica. Por lo tanto, el análisis de la corriente es una herramienta útil para detectar problemas en el motor que provoquen modulaciones periódicas en la corriente. 


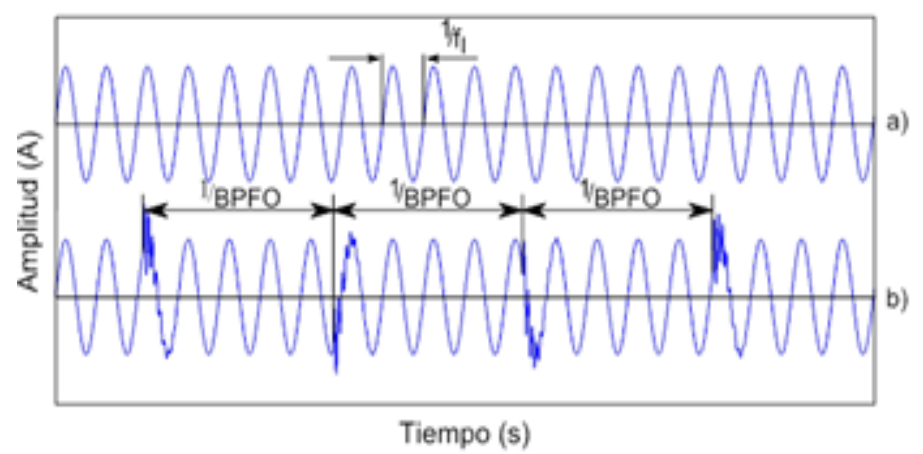

Fig. 1. Formas de onda de las corrientes para una de las fases del motor cuando está sano (a) y con un rodamiento picado en la pista externa (b).

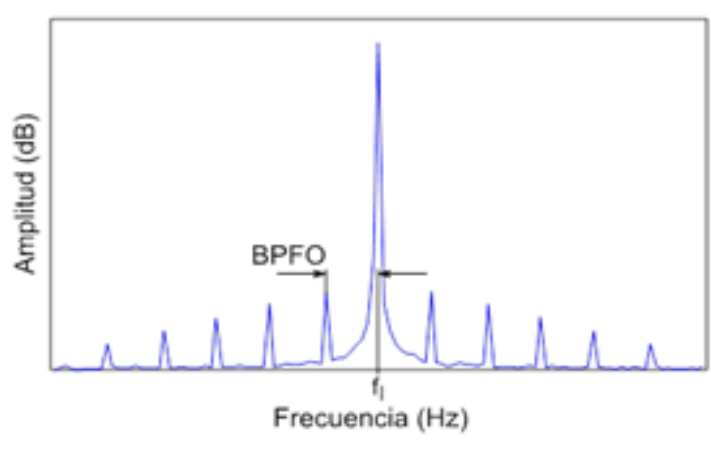

Fig. 2. Espectro de la corriente para el caso b).

Un modelo comúnmente utilizado para analizar problemas eléctricos en el rotor de un motor de inducción es el presentado por Touhami et al. (2006), basado en la teoría de los circuitos acoplados magnéticamente. Este modelo consiste en representar al rotor como una malla de circuitos con resistencias e inductancias que representan cada barra y cada porción de los anillos que las cortocircuitan. Fig. 3 muestra el modelo de un rotor sano y uno con una barra rota en el rotor.

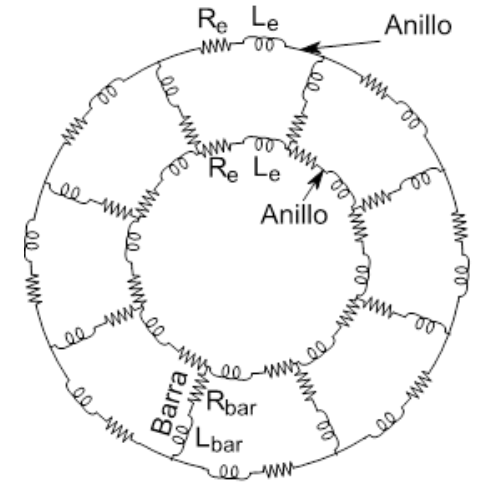

a)

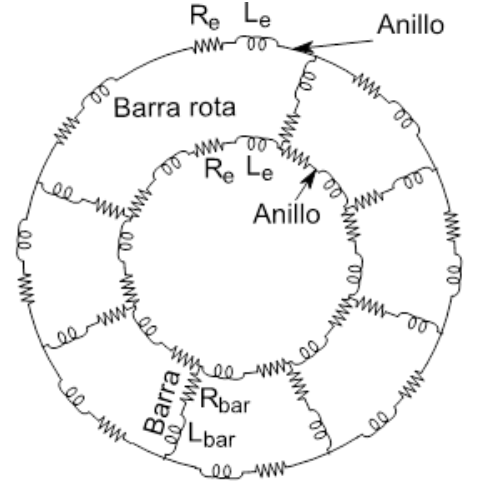

b)

Fig. 3. Modelo de un rotor sano a) y de un rotor con una barra rota b).

El efecto que tiene una barra dañada en el rotor es que la resistencia $R_{b a r}$ y la inductancia $L_{b a r}$, de esa barra en particular serán distintas a las del resto de las barras. En el trabajo de Kliman et al. (1988), se demostró que cuando se consideraba una barra rota en el rotor, eliminando la resistencia y la inductancia que representan esa barra en particular en el modelo, como se muestra en Fig. 3 b), se presentan bandas laterales alrededor de la componente fundamental en el espectro de la corriente. La causa de esto es la modulación periódica en amplitud que se genera en la corriente debido a la presencia de la barra rota. Esta modulación se genera debido a que cada vez que un polo magnético pasa por sobre la barra rota, no se inducen corrientes sobre ella. Esto hace que exista una disminución del par en ese instante y por lo tanto aumente el deslizamiento, lo que hace que aumente la corriente que alimenta al estator. Estas modulaciones ocurren a la frecuencia con la que un polo pasa por sobre una barra en particular, llamada frecuencia de paso de polos, por lo tanto son periódicas.

Por lo tanto, cualquier asimetría que se genere en el campo magnético rotórico, como ser barras o anillos extremos dañados, modularán en amplitud la corriente. El espectro de la corriente presentará entonces bandas laterales alrededor de la componente fundamental de la corriente, separadas a la frecuencia de paso de polos. La frecuencia de paso de polos está dada por la ecuación (1):

$f_{p p}=2 n_{p p} f_{\text {des }}$

\section{Donde:}

$\mathrm{f}_{\mathrm{pp}}=$ Frecuencia de paso de polos

$\mathrm{n}_{\mathrm{pp}} \quad=\quad$ Número de pares de polos

$\mathrm{f}_{\text {des }}=$ Velocidad de deslizamiento 
Debido a la asimetría en la construcción del rotor, se pueden presentar pequeñas bandas laterales a la frecuencia de paso de polos incluso si las barras están en buen estado. Como indica Cabanas (1998) en su trabajo Métodos no convencionales para la detección y diagnostico precoz de fallos en motores eléctricos, existen estándares que evalúan la condición de las barras del motor utilizando la diferencia en decibeles que existe entre la magnitud de la componente fundamental de la corriente y la magnitud de su primera banda lateral izquierda separada a $\mathrm{f}_{\mathrm{p}}$. Diferencias sobre $60[\mathrm{~dB}]$ indican que el estado de las barras es excelente y diferencias menores a $30[\mathrm{~dB}]$ o $40[\mathrm{~dB}]$ indican barras agrietadas o rotas.

\section{ANÁLISIS DE LA VARIACIÓN DE LA VELOCIDAD ANGULAR INSTANTÁNEA (VAI)}

En la práctica, muchas máquinas funcionan a velocidad variable, incluyendo a los motores de inducción. Estas variaciones de velocidad pueden ser inherentes al funcionamiento de la máquina o provenir de fallas en ella. En el presente trabajo se analiza las variaciones de la velocidad angular en el eje para obtener información del estado de las barras del rotor.

El análisis de las variaciones de la velocidad angular instantánea consiste en medir la velocidad angular del eje en cada instante de tiempo y analizar la frecuencia de sus variaciones. Dependiendo del problema que presente el motor, las frecuencias de las variaciones deberán presentar características del problema en particular, lo que permite diagnosticarlo. En su forma diferencial, la velocidad angular del eje es de la forma:

$\omega=\frac{\mathrm{d} \theta}{\mathrm{dt}}$

Donde $\theta$ es el ángulo de giro del eje.

En la práctica, lo que se hace en realidad es medir el tiempo que tarda el eje en efectuar algún desplazamiento angular. Así se llega a que la forma discreta de la velocidad angular en el eje está dada por la ecuación (3):

$\omega=\frac{\Delta \theta}{\Delta \mathrm{t}}$

El desplazamiento angular puede obtenerse de un dispositivo que entregue un pulso cada cierto desplazamiento angular efectuado y luego cuantificar el tiempo que tardó éste. El cociente entre ellos, presentado en la ecuación (3), es la velocidad angular promedio en ese tramo. Como en este caso se requiere la variación de velocidad instantánea en cada revolución, se requiere un dispositivo que entregue una cantidad elevada de pulsos por vuelta, por ejemplo, un encoder. Li et al. (2001) explican de manera más extensa algunos métodos de medición de la velocidad angular. La relación entre el par que actúa sobre el rotor y su velocidad de giro está dada por la ecuación del movimiento:

$\frac{\mathrm{d} \omega}{\mathrm{dt}}=\frac{\mathrm{T}_{\mathrm{M}}-\mathrm{T}_{\mathrm{L}}}{\mathrm{J}}$

Donde:

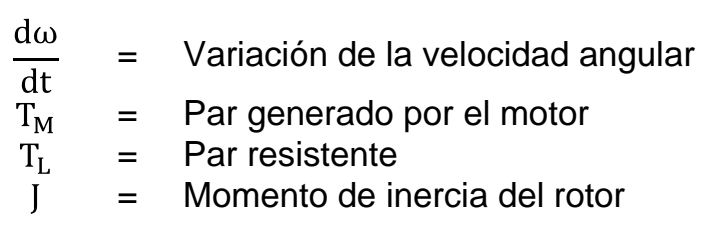

El par resistente depende del proceso en particular y de la condición mecánica del rotor debido a rodamientos dañados y roces parciales, y es independiente de la condición eléctrica del motor. Una barra dañada en el rotor tendrá una resistencia mayor a la que debiera presentar, por lo que cada vez que un polo pasa frente a esta barra, se induce una corriente menor en ella (Chapman, 2000). Al ser menor la corriente en esa barra en particular, el par ejercido sobre el rotor es también menor. Esto genera un par motor que varía periódicamente en amplitud a la $\mathrm{f}_{\mathrm{pp}}$ y por lo tanto, de acuerdo a la ecuación (4), generará variaciones periódicas a $f_{p p}$ en la velocidad de rotación.

En la práctica, el par producido por un motor de inducción no es constante, incluso si no presenta problemas. Existe una fluctuación en el par debido al paso del campo magnético del estator entre una barra 
y la siguiente. De este modo, el par motriz varía periódicamente a la frecuencia con la que el campo magnético del estator corta las barras del rotor.

Como indican Sasi et al. (2006), cuando existen barras dañadas en el rotor de un motor de inducción, se presentan principalmente dos síntomas en el espectro de la VAl: Componentes múltiplos de la frecuencia de paso de polos y bandas laterales alrededor de la componente a la velocidad de giro, separadas a la frecuencia de paso de polos.

El primer síntoma es concordante con lo señalado en este trabajo. El segundo síntoma, observado en las mediciones experimentales, se debe, como se explica en el trabajo de Talbot (2012), a errores en la medición de la VAl y no a la hipótesis usada por Sasi et al. (2006) de que el par resistente varía a la velocidad de rotación, hipótesis que no tiene ningún fundamento físico.

Existen errores geométricos como la separación desigual entre ranuras en la construcción de un encoder, los cuales se repetirán a cada vuelta. Así, se presentarán componentes a la frecuencia de giro, a pesar de que la velocidad no esté variando con esa frecuencia. Por otro lado, el desbalanceo residual del rotor demostró tener influencia sobre la VAI (Talbot, 2012). Por lo tanto, la componente a 1xRPM encontrada en el espectro de la VAI descrita por Sasi et al. (2006) no es generada por las causas que los autores indican, sin embargo las bandas laterales alrededor de las componentes múltiplos de la frecuencia de giro del rotor, separadas a la frecuencia de paso de polos, sí son generadas por las barras rotas.

En este trabajo sólo se considerará el síntoma que corresponde a las componentes a la frecuencia de paso de polos, ya que si bien las bandas laterales a la frecuencia de paso de polos encontradas alrededor de la componente a la velocidad de giro son producto del daño en las barras, también son producto de la modulación de un error que depende del instrumento que se utilice y del valor del desbalanceo residual que depende de cada rotor en particular. Si este error o el desbalanceo no existieran o fueran muy pequeños, las bandas laterales no estarían presentes en las mediciones.

Debido a asimetrías en la construcción del rotor, los campos magnéticos inducidos en éste nunca serán del todo simétricos. Por lo tanto, estos síntomas podrán presentarse incluso cuando el rotor está sano, sólo que la magnitud de las componentes será bastante menor.

\section{ANÁLISIS DE LAS VIBRACIONES}

El análisis de las vibraciones es una técnica muy utilizada en el mantenimiento predictivo. Consiste principalmente en medir las vibraciones de la máquina mediante un acelerómetro y luego procesar la señal mediante distintas técnicas para evaluar si la máquina presenta algún tipo de falla.

Debido a la disminución en la corriente que circula por una barra dañada, el efecto provocado sobre el motor es muy similar al que ocurre si el entrehierro en ese punto fuese mayor, como se ilustra en Fig. 4, debido a que la fuerza radial entre ese punto del rotor y el estator es menor. En este caso, la dirección radial en la cual se produce esta diferencia en la fuerza radial gira con el rotor a la velocidad de giro, por lo que se genera una fuerza desbalanceada rotatoria a 1XRPM y en consecuencia, vibraciones periódicas a la velocidad de rotación del motor. Esta fuerza actuará sobre el rotor de la misma manera en que lo hace el desbalanceo, una fuerza de valor constante que gira con el rotor.

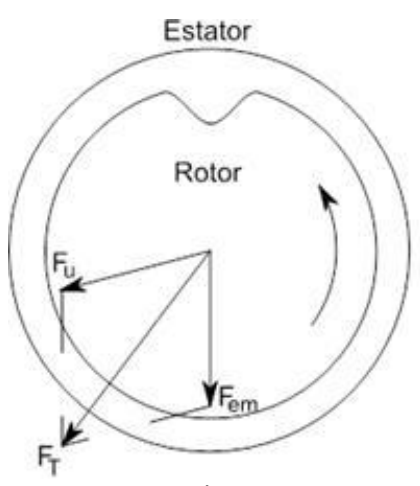

a)

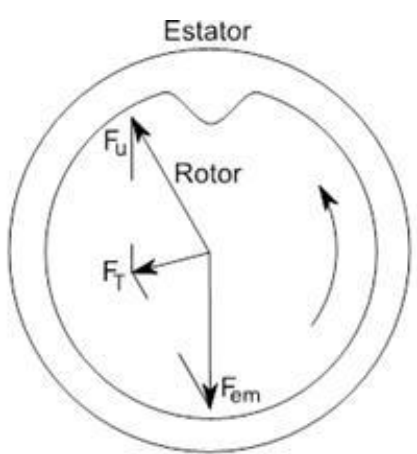

b)

Fig. 4. Fuerza resultante $\mathrm{F}_{\mathrm{T}}$ debido a la interacción entre la fuerza electromagnética desbalanceada $\mathrm{F}_{\mathrm{em}}$ y la fuerza $\mathrm{F}_{\mathrm{u}}$ generada por el desbalanceamiento. 
Tanto la fuerza electromagnética desbalanceada $\mathrm{F}_{\mathrm{em}}$, como la fuerza generada por el desbalanceamiento $\mathrm{F}_{\mathrm{u}}$, giran a la velocidad de rotación, por lo que ambas se suman para dar una fuerza resultante $\mathrm{F}_{\mathrm{T}}$ en el rotor, pudiendo tanto aumentar como disminuir el valor de las vibraciones, como se ilustra en Fig. 4. A diferencia del desbalanceo, la fuerza electromagnética desbalanceada no es de valor constante. Cada vez que la barra rota se alinea con uno de los polos del estator, se genera un máximo para esta fuerza, por lo que esta fuerza y por lo tanto las vibraciones a 1xRPM son moduladas a la frecuencia de paso de polos. De este modo, en el espectro se presentarán bandas laterales alrededor de la componente a la velocidad de giro separadas a la frecuencia de paso de polos.

\section{ENSAYO EXPERIMENTAL: BARRAS CON AUMENTO DE DAÑO}

A modo de validar los modelos teóricos, se realizó un ensayo experimental que consistió en analizar los síntomas que genera un motor al que se le daña una barra de manera progresiva. Se evalúa experimentalmente cuál de los tres métodos mencionados permite identificar de manera más incipiente el daño en las barras del motor. El ensayo experimental consistió en dañar progresivamente una barra en el rotor y aplicar las tres técnicas de diagnóstico para distintas cargas. El banco de ensayos está compuesto por un motor asíncrono de dos pares de polos cuya corriente nominal es de 13,6 [A] y su potencia de 9,2 $[\mathrm{Kw}]$ en el que se medirán las vibraciones, la corriente en una de las fases que alimentan al estator y la VAI del eje según el montaje mostrado en Fig. 5.

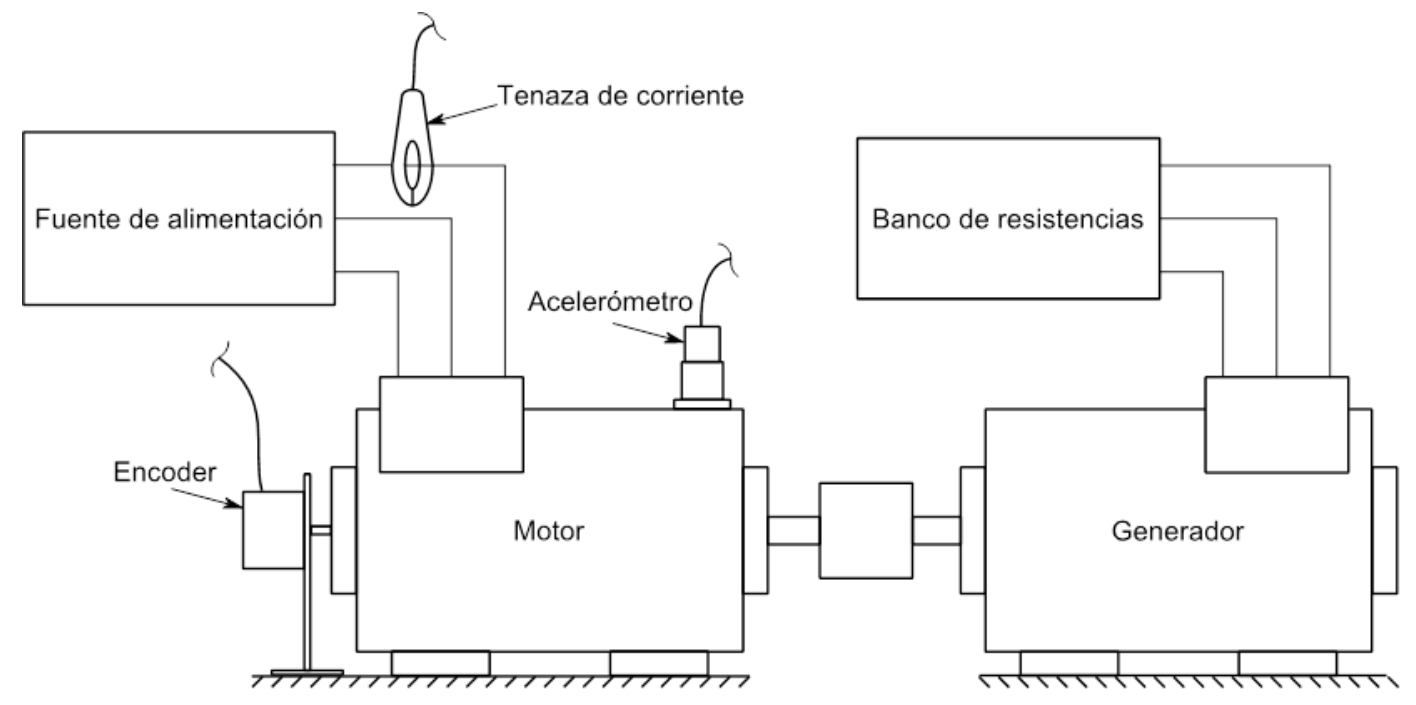

Fig. 5. Esquema del banco de ensayos utilizado para la experiencia.

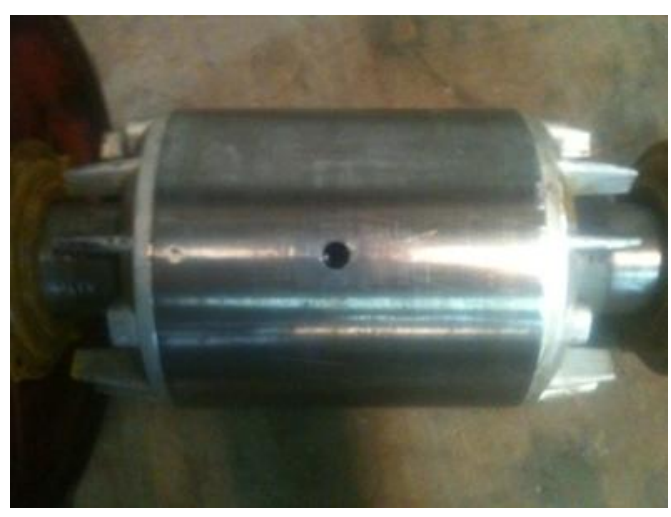

Fig. 6. Perforación en una de las barras del rotor.

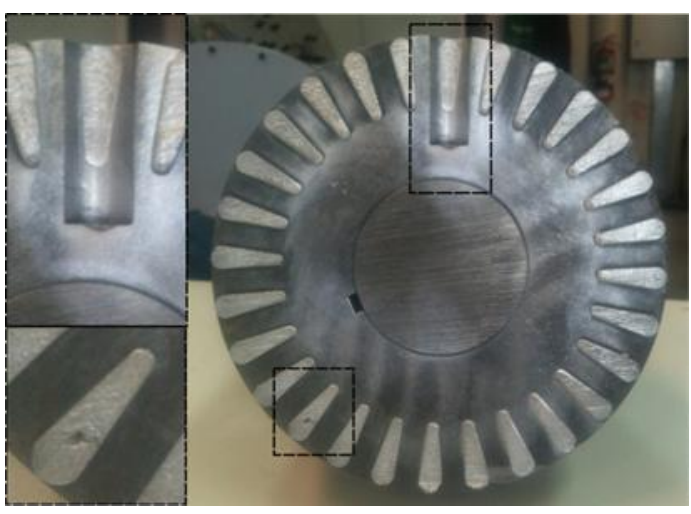

Fig. 7. Corte transversal del rotor.

El porcentaje de carga del motor se consideró como el porcentaje de la corriente nominal que alimenta el motor para cada caso. El daño en una de las barras del rotor se generó haciendo una perforación de 6 [mm] de profundidad y $10[\mathrm{~mm}]$ de diámetro sobre una barra en primera instancia para luego ir avanzando 3 [mm] de profundidad cada vez hasta llegar a cortar la barra por completo, como muestra Fig. 6 y Fig. 7. El porcentaje de daño en la barra se calcula considerando el porcentaje del área transversal de la barra removida según la profundidad de la perforación. 


\section{ANÁLISIS DE LA CORRIENTE}

La Fig. 8 muestra tres espectros de la corriente para una carga de $85 \%$ de la corriente nominal y tres niveles distintos de daño en una barra. El espectro de la corriente para el estado inicial del motor no presenta síntomas de barras rotas, mientras que a medida que el daño en la barra aumenta, las bandas laterales alrededor de la componente fundamental de la corriente a $50[\mathrm{~Hz}]$ comienzan a distinguirse y son más notorias, aumentando su magnitud. La Fig. 9 muestra tres espectros de la corriente para una barra rota con el motor funcionando a distintas cargas. Se observa que a medida que la carga aumenta, la magnitud de las bandas laterales a $\mathrm{f}_{\mathrm{pp}}$ también aumenta.

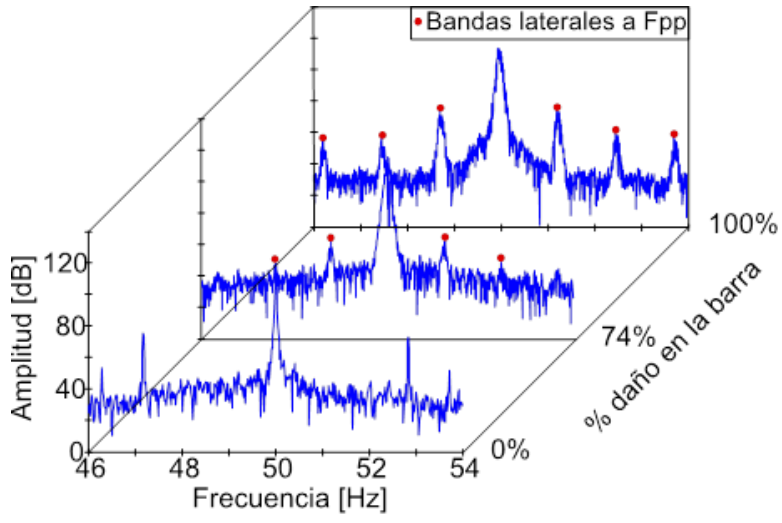

Fig. 8. Espectros de corriente para tres niveles de daño y una carga de $85 \%$.

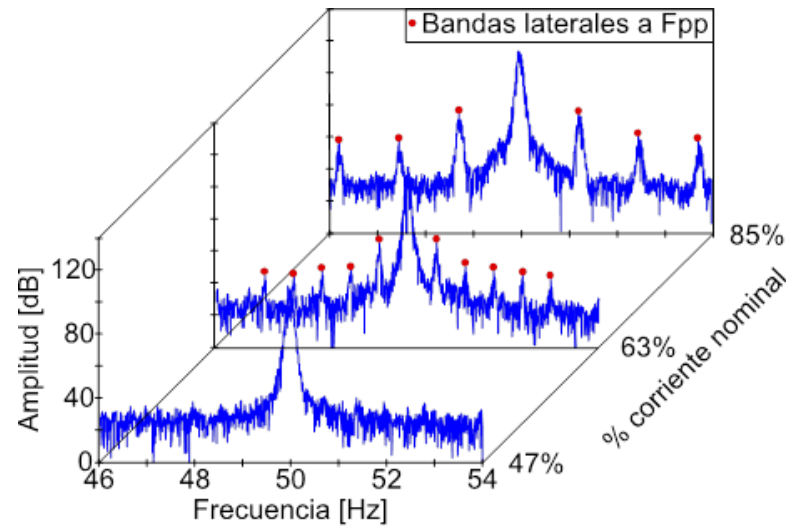

Fig. 9. Espectros de corriente para tres niveles de carga y una barra cortada.

Se puede observar para este caso que cuando el daño en la barra es muy pequeño o cuando el porcentaje de la corriente nominal que alimenta al motor está bajo el $50 \%$, incluso existiendo una barra cortada, el método de la corriente no es capaz de detectar el problema. Los estándares existentes para evaluar la severidad del daño en barras mediante la el análisis de la corriente utilizan la diferencia en decibeles entre la amplitud de la componente fundamental de la corriente y su primera banda lateral izquierda. Este valor obtiene por la ecuación (5):

$\mathrm{R}_{\mathrm{b}}=20 \log \frac{\mathrm{F}_{\mathrm{L}}}{\mathrm{F}_{\mathrm{P}}}$

\section{Donde:}

$\mathrm{F}_{\mathrm{p}}=$ Amplitud de la primera banda lateral a la frecuencia de paso de polos a la izquierda de la frecuencia de la línea

$\mathrm{F}_{\mathrm{L}}=$ Amplitud de la componente a la frecuencia de la línea

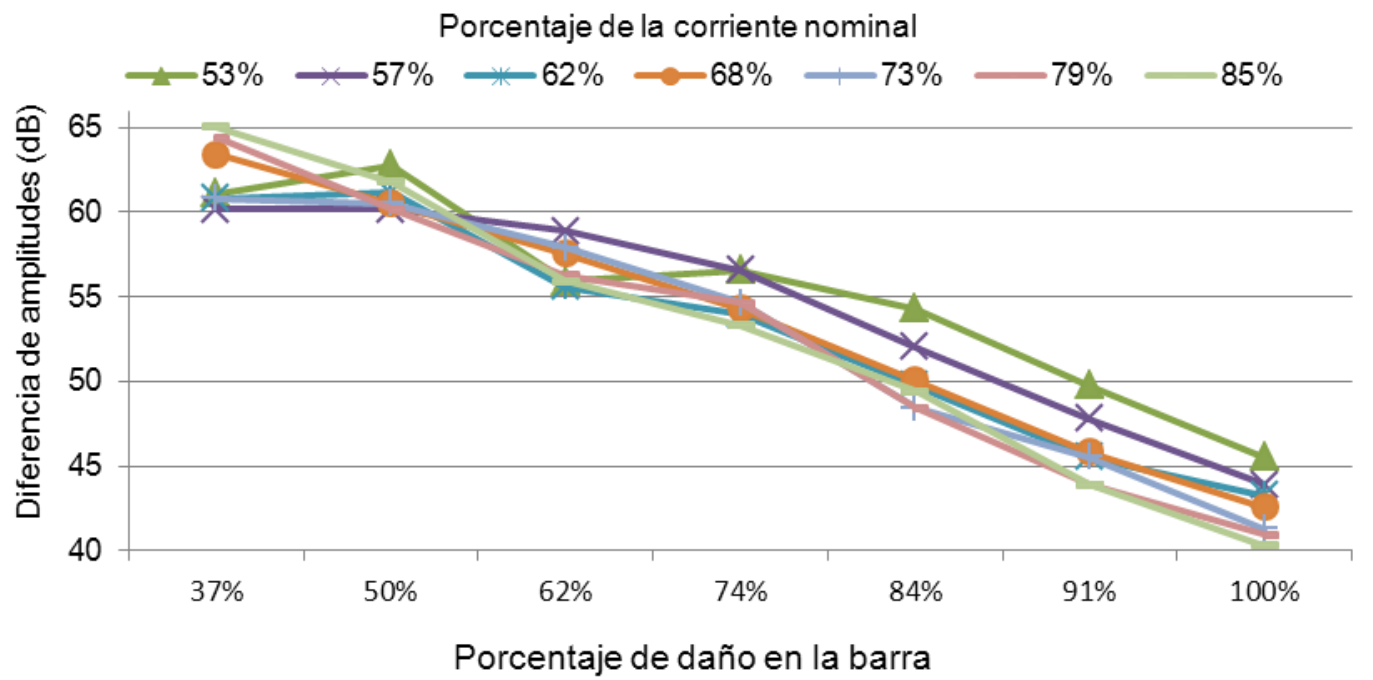

Fig. 10. Valores del parámetro $\mathrm{R}_{\mathrm{b}}$ para cada carga en particular conforme aumenta el daño en una barra. 
Los estándares de evaluación existentes son para motores de más de 40 [Hp], potencia que está por sobre la potencia del motor utilizado en este ensayo. De cualquier modo, el valor de $\mathrm{R}_{\mathrm{b}}$ no debiera cambiar su comportamiento para motores de potencia más pequeña, sólo que por su valor no podrá utilizarse los estándares para evaluar la condición de las barras del rotor. El gráfico de Fig. 10 muestra cómo cambia $\mathrm{R}_{\mathrm{b}} \mathrm{a}$ medida que aumenta el daño en la barra para cada nivel de carga. Se observa que el parámetro $\mathrm{R}_{\mathrm{b}}$ disminuye su valor conforme aumenta la carga en el motor.

\section{MÉTODO DE LA VELOCIDAD ANGULAR INSTANTÁNEA (VAI)}

Fig. 11 muestra tres espectros en cascada de la VAI para un $85 \%$ de la carga nominal del motor y distintos niveles de daño en la barra. Se puede observar que para una carga constante en el motor, la magnitud de las componentes a múltiplos de la frecuencia de paso de polos aumenta conforme aumenta el daño en la barra.

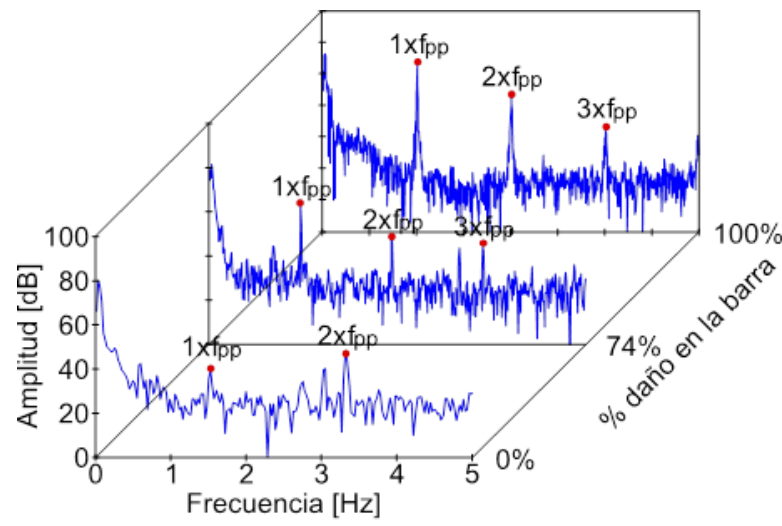

Fig. 11. Espectros de la VAI para un $85 \%$ de la corriente nominal y tres niveles de daño en la barra.

En el espectro de la VAI para la condición inicial del motor se pueden identificar componentes a la frecuencia de paso de polos. Si bien el motor aún no había sido intervenido en este punto, esto sugiere la existencia de un daño previo en las barras del rotor. Se hizo un corte transversal del rotor, como se aprecia en Fig. 7, en el que se observaron algunas imperfecciones en las barras. En ella se puede identificar una porosidad en una de las barras, lo que representa una disminución en el área transversal en ese punto y por lo tanto una mayor resistencia. Para este caso, la técnica de la VAl fue más sensible que el del análisis de la corriente para diagnosticar daño incipiente en las barras, ya que fue capaz de detectar imperfecciones tan pequeñas como porosidades en las barras. Tanto la técnica basada en el análisis de la corriente como la técnica basada en las vibraciones fueron incapaces de detectar esta imperfección. A diferencia de lo que sucede en el caso del análisis de la corriente, no existen estándares para evaluar el estado de las barras del rotor mediante el análisis de la VAI en el eje.

Como ya se mencionó, en el análisis de la corriente se utiliza para evaluar el estado de las barras del rotor la relación $\mathrm{R}_{\mathrm{b}}$. De manera análoga, en este trabajo se definirá el parámetro $\mathrm{R}_{\mathrm{E}}$, expresado en decibeles, como muestra la ecuación (6):

$\mathrm{R}_{\mathrm{E}}=20 \log \frac{\mathrm{F}_{\mathrm{R}}}{\mathrm{F}_{\mathrm{P}}}$

Donde:

$\mathrm{F}_{\mathrm{P}} \quad=\quad$ Amplitud de la componente a la frecuencia de paso de polos

$\mathrm{F}_{\mathrm{R}}=$ Velocidad de rotación del motor

El parámetro $R_{E}$ representa la diferencia en decibeles que existe entre la velocidad de rotación del motor y la magnitud de la primera componente a la frecuencia de paso de polos en el espectro de la VAl. El grafico de Fig. 12 muestra el valor de $\mathrm{R}_{\mathrm{E}}$ para diferentes niveles de carga y distintos niveles de daño en la barra. Se puede ver que, al igual que en el caso del análisis de la corriente, la tendencia de $\mathrm{R}_{\mathrm{E}}$ es disminuir su valor conforme el daño aumenta. 


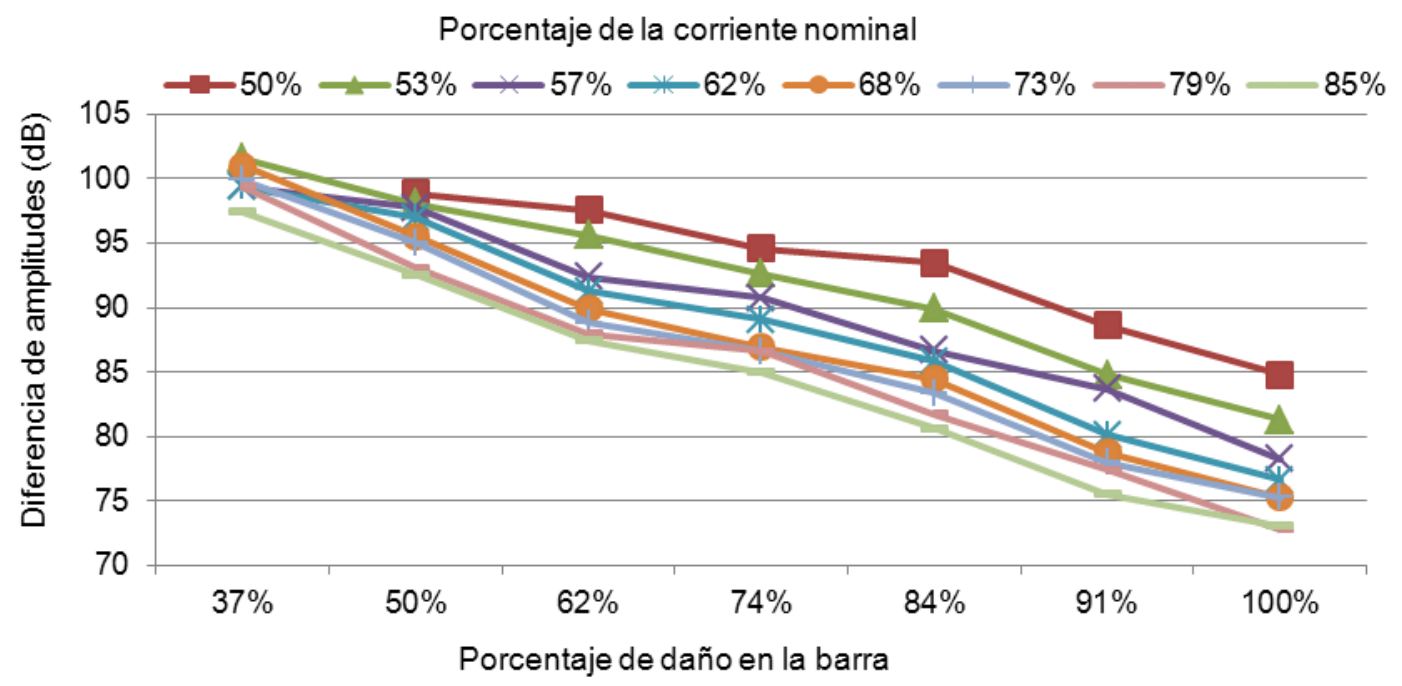

Fig. 12. Valor del $R_{E}$ para los distintos niveles de daño y una carga en particular.

Considerando que existe una relación entre el parámetro $R_{E}$ y el nivel de daño en las barras del motor, es de esperar que el análisis de la VAI pueda ser utilizado como un método de diagnóstico eficiente para evaluar la condición de las barras en el motor. Sin embargo, deberán hacerse más estudios al respecto para determinar qué valores deberán considerarse para determinar el nivel de daño en las barras y eventualmente crear un estándar que pueda ser aplicado a casos generales.

\section{ANÁLISIS DE VIBRACIONES}

Fig. 13 muestra tres espectros obtenidos en el ensayo para una carga constante y distintos niveles de daño. Se observa que las bandas laterales alrededor de la 1xRPM, síntoma característico del problema, se presentan cuando el daño en la barra ya está en un estado más avanzado, alrededor de un $74 \%$. Fig. 14 muestra tres espectros de vibraciones para una barra cortada con distintos niveles de carga. Este método mostró no ser tan incipiente como los otros dos, por lo tanto se escogió el caso de la barra completamente cortada para apreciar mejor los síntomas.

Se debe tener presente que al perforar la barra para dañarla se generan dos efectos: Una fuerza electromagnética desbalanceada rotatoria con la velocidad de rotación y una fuerza centrífuga rotatoria debido al desbalanceo que genera la perforación en la barra. No se puede utilizar entonces las variaciones en el valor de la componente a 1xRPM para diagnosticar el daño en las barras del rotor para este caso, pero si se puede usar el valor de las bandas laterales a $\mathrm{f}_{\mathrm{pp}}$.

Como el valor de las bandas laterales a la frecuencia de paso de polos es de muy bajo valor, es posible que la dispersión en el valor de la frecuencia que generará una leve variación de velocidad de rotación no permita discriminarlas del ruido de fondo de la medición. Este hecho se ve en algunas mediciones, sobre todo en las de cargas más bajas.

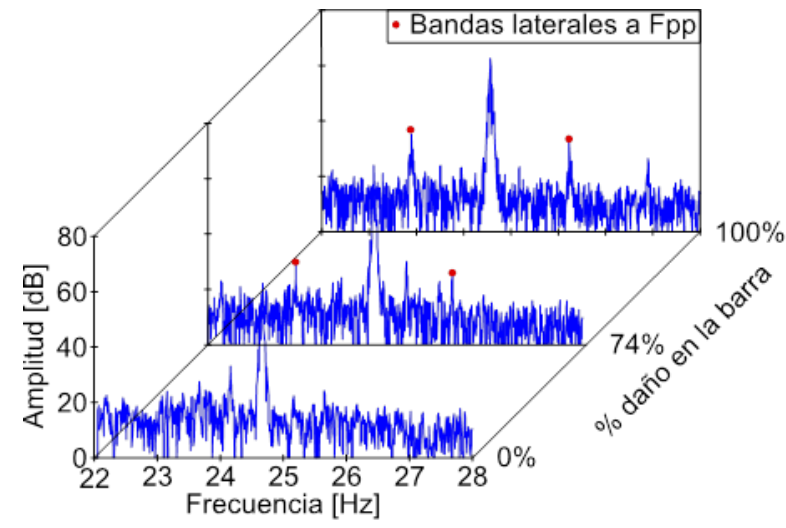

Fig. 13. Espectros de las vibraciones para un $85 \%$ de la corriente nominal y distintos niveles de daño en la barra.

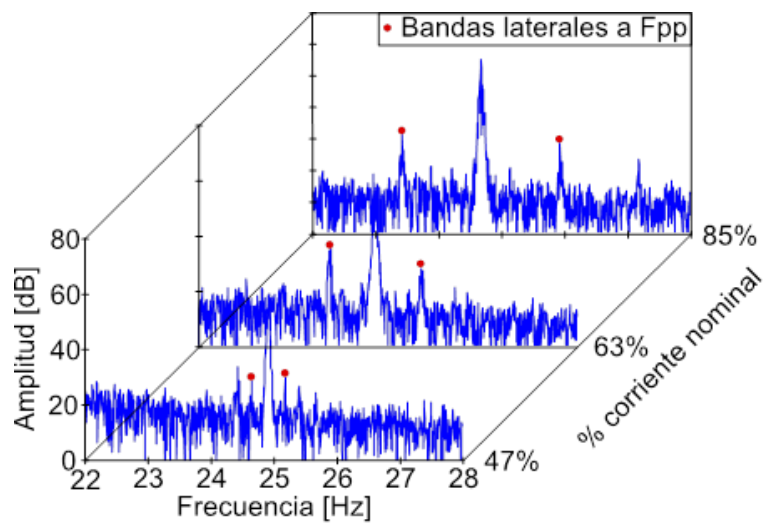

Fig. 14. Espectros de las vibraciones para un 100\% de daño en la barra y distintas cargas para el motor. 
Se concluye que el análisis de las vibraciones es un método que permite diagnosticar la presencia de barras dañadas en el rotor de un motor de inducción, siempre y cuando el daño en las barras sea avanzado. No es adecuado para la determinación incipiente de daño en las barras

\section{CONCLUSIONES}

Es posible diagnosticar barras dañadas en el rotor de un motor de inducción mediante cualquiera de los tres métodos analizados en este estudio. De los ensayos experimentales, se concluye que para este caso tanto el análisis de la VAI como el análisis de la corriente fueron capaces de detectar daño incipiente en una de las barras del rotor. El análisis de la VAI pudo identificar asimetrías en el rotor, tales como porosidades en las barras.

La técnica del análisis de las vibraciones demostró requerir mayores niveles de daño en las barras para diagnosticar el problema en relación a las otras dos técnicas. No se encontró una relación entre las vibraciones y el nivel de daño en las barras o el nivel de carga en el motor, por lo tanto, se concluye que, si bien este método mostró ser una herramienta que permite diagnosticar el problema de las barras dañadas, no es útil para predecir el nivel de daño que existe en las barras del motor.

Los errores geométricos que presentan los encoders generan señales periódicas que se repiten a cada vuelta, lo que introduce componentes a 1xRPM en el espectro de la VAl que no son reales. Además, el desbalanceo residual afectará la $\mathrm{VAI}$, haciéndola variar periódicamente a cada vuelta, lo que también introduce componentes a 1xRPM.

A futuro, la técnica basada en el monitoreo de la VAI podría constituir un método para evaluar la condición de las barras del motor, debido a que existe una relación entre el nivel de daño en las barras y la magnitud de los síntomas. Para ello, se deberán efectuar más estudios al respecto considerando más factores, tales como el tamaño y potencia de la máquina o la geometría del rotor. Estos estudios permitirán crear estándares para evaluar la condición de las barras del motor, similares a los existentes en el análisis de la corriente.

\section{REFERENCIAS}

Chapman S.J., Máquinas eléctricas, $3^{\text {era }}$ edición, McGraw Hill. (2000)

Kliman G.B., Stein J., Endicott R.D., Noninvasive detection of broken rotor bars in operating induction motors, IEEE Transactions on energy conversion, vol. 3, no. 4, december (1988)

Li Y., Gu F., Harris G., Ball A., Bennett N., Travis K., The measurement of instantaneous angular speed, Mechanical Systems and Signal Processing 19, 786-805 (2005)

Nandi, S., Toliyat H. A., Condition Monitoring and Fault Diagnosis of Electrical Machines - A Review, Industry Applications Conference, Thirty-Fourth IAS Annual Meeting. Vol. 1, 197 - 204 (1999)

Roque, A.A, Silva T.A.N., Calado, J.M.F., Dias J.C.Q., Rolling bearing fault detection and isolation - A didactic study, 4th wseas/iasme, International Conference On Educational Technologies (EDUTE'08)

Corfu, Greece, October 26-28, (2008)

Sasi, A.Y.B., Gu F., Li Y., Ball A.D, A validated model for the prediction of rotor bar failure in squirrel-cage motors using instantaneous angular speed, Mechanical Systems and Signal Processing 20, 1572-1589 (2006)

Szabó L., Dobai J.B., Biró K.Á., Rotor Faults Detections in Squirrel-Cage Induction Motors by Current Signature Analysis, International Conference on Automation, Quality and Testing, Robotics, Cluj-Napoca, Romania, May 13 - 15, (2004)

Talbot, C., Diagnóstico de problemas críticos en motores de inducción, Tesis de Magister, Departamento de Ingeniería Mecánica, Universidad de Concepción, Concepción, Chile (2012)

Touhami O., Noureddine L., Ibtiouen R., Fadel M., Modeling of the Induction Machine for the Diagnosis of Rotor Defects. Part. I: An Approach of Magnetically Coupled Multiple Circuits, Industrial Electronics Society, 31st Annual Conference of IEEE, 6-10 November (2005) 